Methods Retrospective review of clinical records and diagnostic tests.

Results A 78-year-old man with cerebral and lung melanoma metastases was treated with nivolumab, the newly developed monoclonal antibody which inhibits PD-1 deactivation of Tcells. He showed good radiological response.

Three months after ceasing nivolumab, he presented to ED with acute confusion, aphasia, left-sided weakness and neglect as a stroke call. MRI demonstrated no evidence of stroke or acute encephalitis. EEG showed diffuse slowing. Lumbar puncture was not performed as he was therapeutically anticoagulated and had begun to improve on empirical antibiotics and acyclovir. One week later, he suddenly deteriorated with fevers and reduced level of consciousness. Acyclovir was restarted. Repeat EEG revealed periodic epileptic discharges over the right hemisphere and MRI demonstrated hyperintense signal and diffusion restriction of the right mesial temporal lobe suggestive of encephalitis. Subsequent lumbar puncture was negative for HSV, VZV, enterovirus, and autoimmune encephalitis antibodies.

The patient's oncology team had been in support of a course of methylprednisone on suspicion of autoimmune encephalitis, but by the time the lumbar puncture results returned he was again improving. A diagnosis of suspected nivolumabinduced encephalitis was made based on exclusion of other possible diagnoses.

Conclusions The rising use of immune checkpoint inhibitors heralds an array of new and unexpected neurological complications including autoimmune encephalitis. A nationwide registry of possible cases may be helpful in improving our currently limited understanding of these emerging complications.

\section{COGNITIVE IMPAIRMENT IN THE COMMUNITY: A 16- MONTH AUDIT STUDY OF A NEUROLOGY MEMORY CLINIC IN THE INNER SYDNEY REGION}

${ }^{1}$ Louise Rigney, ${ }^{2}$ Lily Chen, ${ }^{3}$ Alexis Selby, ${ }^{4}$ Tejas Patel, ${ }^{5}$ Yun T Hwang, ${ }^{2}$ Anthony ED Mobbs, ${ }^{2,6,7}$ Rowena EA Mobbs*. 'Department of Neurology, Campbelltown Hospital, Campbelltown, NSW, Australia, Australia; ${ }^{2}$ Sydney Cognitive, North Sydney, NSW, Australia; ${ }^{3}$ Department of Neurology, Northern Beaches Hospital, Frenchs Forest, NSW, Australia; ${ }^{4}$ Department of Neurology, Bankstown Hospital, Bankstown, NSW, Australia; ${ }^{5}$ Brain and Mind Centre, University of Sydney, Camperdown, NSW, Australia; ${ }^{6}$ University of Sydney, Camperdown, NSW, Australia; ${ }^{7}$ Macquarie University, Macquarie, NSW, Australia

10.1136/jnnp-2019-anzan.101

Introduction Neurodegenerative disease is increasingly common, but neurology service utilisation by this population is unclear. This study presents an integrated metropolitan neurology clinic as a model of care, and examines care pathways and characteristics in patients with cognitive decline.

Methods An audit of patients with dementia ( $n=136,51-96$ yr,M:F1.1:1) and mild cognitive impairment (MCI) $(n=28,56-$ 83 yr,M:F0.6:1) over a 16 month period was performed using retrospective analysis of demographic characteristics, clinical information and frailty measures.

Results The average duration of cognitive decline at presentation was 2.7 years in dementia $(\mathrm{n}=135, \mathrm{M}=3.1, \mathrm{~F}=2.3)$ and 1.5 years MCI $(\mathrm{n}=28, \mathrm{M}=1.7, \mathrm{~F}=1.3)$. Average age of patients with newly diagnosed dementia was 72 (M:F0.96:1) and MCI 70 (M:F1.03:1) with Alzheimer's disease in 86(64\%) dementia with Lewy bodies $13(10 \%)$ frontotemporal dementia $11(8 \%)$ and distinct overlap syndromes in $7(5 \%)$.
Depression was associated with younger age at diagnosis (ave.60) versus anxiety(74) and agitation(75). Psychosis was in 14(10\%) REM sleep behaviour disorder 6(5\%). In the 12 months prior, falls were seen in $30(22 \%)$ and weight loss 29 (21\%). Allied health input occurred in $81 \%$ of dementia versus $68 \%$ MCI cases.

Conclusions Delay in diagnosis from first clinical manifestation, high rate of psychological and medical comorbidity, presence of overlap syndromes, and allied health requirement, support the need for integrated neurological services in dementia.

\section{IS MBS RESTRICTION ON EEGS TRULY BENEFICIAL? RETROSPECTIVE OBSERVATIONAL ANALYSIS OF EEG REFERRAL PATTERNS}

${ }^{1}$ Chathri Ratnayake*, ${ }^{1}$ Ramja Kokulan, ${ }^{1,2}$ Patrick Carney. 'Department of Neurology, Eastern Health, Box Hill, VIC, Australia; ${ }^{2}$ Eastern Health Clinical School, Faculty of Medicine Dentistry and Health Sciences, Monash University, Box Hill, VIC, Australia

\subsection{6/jnnp-2019-anzan.102}

Introduction The November 2018 Medicare Benefits Schedule (MBS) Taskforce draft report recommends standardised national referral form for routine electroencephalogram (EEG) requests to prevent low value clinician referrals. MBS defines twelve clinical presentations where routine EEG is of relatively low diagnostic value. We aim to identify proportion of MBS defined low diagnostic value EEGs and likely referral patterns. Methods Retrospective single centre observational study was conducted from January to December 2018. All EEG referrals to a tertiary hospital neurodiagnostic unit were categorised as low or high yield based on MBS recommendation. Sub-group analysis of the low yield group was carried out.

Results Total of 1210 EEG referrals were analysed and 5 were excluded from analysis due to insufficient clinical data. Of these referrals 1114 (92.4\%) were for high yield indications, $77(6.4 \%)$ for low yield and $14(1.2 \%)$ were indeterminate as to low or high value. Of low yield referrals, $70 \%$ were referred for syncope or presyncope and 18.2\% for psychogenic nonepileptic seizures. Low yield EEGs were referred by hospital doctors, neurologists and general partitioners by proportions of $37.7 \%, 33.7 \%$ and $28.6 \%$ respectively. Four (5.2\%) low diagnostic value EEGs were abnormal.

Conclusion Current clinical practice for EEG referrals is in line with MBS draft review recommendations. We believe addition of a standardised referral form and restricting referrals will have minimal impact on referral quality.

\section{ALTERED PERIPHERAL AXONAL PROPERTIES IN FIBROMYALGIA}

${ }^{1,2,3}$ Cindy (Shin-YI) Lin*, ${ }^{4}$ Hao-Wen Teng, ${ }^{4,3}$ Jowy Tani, ${ }^{4}$ Hung-Ju Chen, ${ }^{4}{ }^{4} \mathrm{i}-$ Chen Lin, ${ }^{4}$ TsuiSan Chang, ${ }^{4}$ Jia-Ying Sung. 'University of Sydney, Sydney, NSW, Australia; ${ }^{2}$ Central Clinical School, Brain and Mind Centre, University of Sydney, Sydney, NSW, Australia; ${ }^{3}$ Neural Regenerative Medicine, College of Medical Science and Technology, Taipei Medical University, Taipei, Taiwan; ${ }^{4}$ Department of Neurology, Wan Fang Hospital, Taipei Medical University, Taipei, Taiwan

\subsection{6/jnnp-2019-anzan.103}

Introduction Fibromyalgia (FM), a common chronic widespread pain syndrome with neurological symptoms affecting $\sim 10 \%$ of the population worldwide. Whereas small fibre 
neuropathy is a recognized part of FM, surprisingly little is known about large fibre neuropathy. The present study investigates the sensory and motor axonal properties using novel nerve excitability testing (NET) to seek a better understanding of the pathogenesis of this painful disorder.

Methods 25 FM patients were recruited from the Wan Fang Hospital in Taiwan who fulfilled the American College of Rheumatology diagnostic criteria. ${ }^{1}$ NCS, pain scores, blood tests and NET were performed in all patients and patients with factors that may confound the results of NET were excluded. Control data were obtained from age and gendermatched healthy controls (HC) who had no neurological deficits or known pain disorders.

Results The FM group showed an increase in superexcitability $(\mathrm{p}<0.05)$, subexcitability $(\mathrm{p}<0.05)$ and over-shoot during hyperpolarizing threshold electrotonus $(\mathrm{p}<0.05)$ in the sensory excitability profiles in contrast to HC. However, motor nerve excitability profiles showed no significant difference.

Conclusions Alterations in the sensory axonal parameters can be detected while NCS is normal, these findings are compatible with the concept that the sensory system is mainly involved in the pathogenesis of FM. Results implied probable hypofunction of the paranodal fast $\mathrm{K}+$ channel in the sensory axons, known to be associated with the generation of pain. ${ }^{2}$ Our study highlights the advantage of NET over NCS, in the early detection of axonal dysfunction and may provide further understandings of future therapeutic treatment.

\section{REFERENCES}

1. Wolfe F, Clauw DJ, Fitzcharles MA, Goldenberg DL, Hauser W, Katz RS, Mease P, Russell AS, Russell IJ, Winfield JB. Fibromyalgia criteria and severity scales for clinical and epidemiological studies: a modification of the ACR Preliminary Diagnostic Criteria for Fibromyalgia. J Rheumatol 2011;38(6):1113-1122.

2. Kuwabara S, Misawa S. Pharmacologic intervention in axonal excitability: in vivo assessment of nodal persistent sodium currents in human neuropathies. Curr Mol Pharmacol 2008;1(1):61-67.

\section{EMPLOYMENT-ACTIVITY STATUS AND MULTIDISCIPLINARY CARE ENGAGEMENT IN PATIENTS WITH NEWLY DIAGNOSED DEMENTIA: A 16-MONTH AUDIT STUDY WITHIN AN INNER SYDNEY COMMUNITY NEUROLOGY CLINIC}

${ }^{1}$ Louise Rigney, ${ }^{2}$ Alexis Selby, ${ }^{3}$ Lily Chen, ${ }^{4}$ Tejas Patel, ${ }^{5}$ Yun T Hwang, ${ }^{3}$ Anthony ED Mobbs, ${ }^{3,6,7}$ Rowena EA Mobbs*. 'Department of Neurology, Campbelltown Hospital, Campbelltown, NNSW, Australia; 'Department of Neurology, Northern Beaches Hospital, Frenchs Forest, NSW, Australia; ${ }^{3}$ Sydney Cognitive, North Sydney, NSW, Australia; ${ }^{4}$ Department of Neurology, Bankstown Hospital, Bankstown, NSW, Australia; ${ }^{5}$ Brain and Mind Centre, University of Sydney, Camperdown, NSW, Australia; ${ }^{6}$ University of Sydney, Camperdown, NSW, Australia; ${ }^{7}$ Macquarie University, Macquarie, NSW, Australia

\subsection{6/jnnp-2019-anzan.104}

Introduction Dementia is one of the leading causes of mortality and morbidity in Australia. Attitudes towards dementia in the workplace, tailored adjustments for disability, and patientcentred 'exit with dignity' strategies are of objective and subjective importance to patient wellbeing. This study aimed to assess employment characteristics in those with newly diagnosed dementia, and engagement with multidisciplinary supports.

Methods An audit of patients with diagnosis of dementia ( $n=136$,age51-96 yrs,M:F1.1:1) and mild cognitive impairment $(\mathrm{MCI})(\mathrm{n}=28$,age 56-83M:F0.6:1) over a 16 month period in 2017-18 was performed using online server data collection and retrospective analysis of general and employment demographic characteristics, presenting clinical information, and care across clinical psychology, psychotherapy, occupational therapy, speech pathology, and dietetics.

Results Younger onset dementia was present in $14(10 \%)$. Of the 122 dementia cases aged above 65 years, 24(20\%) were employed-active, 98(80\%) were retired, and none were unemployed. Approximately 5\% had a background in healthcare. Allied health support was provided in 106 cases (78\%) with $\geq 3$ supports in $28(21 \%)$ and was more common in those who were retired(76\%) versus employed-active (21\%). Clinical psychology or psychotherapy support was provided in 50 (37\%) cases of dementia.

Conclusions The onset of dementia often co-exists with active employment. Community perception of employment status in dementia would be of future research interest. Provision of multidisciplinary allied health supports in dementia may facilitate coping, adjustment and cooperative strategies for exit with dignity but further studies are required in this cohort.

\section{CASE SERIES: SUBACUTE COMBINED DEGENERATION OF THE SPINAL CORD IN VITAMIN B12-REPLETE RECREATIONAL NITROUS OXIDE ABUSERS}

${ }^{1}$ Chris Blair*, ${ }^{2}$ Chris Tremonti, ${ }^{1}$ Leon Edwards, ${ }^{2}$ Paul Haber, ${ }^{3}$ Michael Halmagyi. ${ }^{1}$ Department of Neurology, Liverpool Hospital, Sydney, NSW, Australia; ${ }^{2}$ Department of Drug Health, Royal Prince Alfred Hospital, Sydney, NSW, Australia; ${ }^{3}$ Department of Neurology, Royal Prince Alfred Hospital, Sydney, NSW, Australia

\subsection{6/jnnp-2019-anzan. 105}

Introduction Within a few years of its discovery in late $18^{\text {th }}$ century, nitrous oxide was being used recreationally for its pleasurable effects. It remains in widespread use as an inhaled stimulant today, and can be legally acquired in bulk quantities with relative ease. In the body prolonged exposure to nitrous oxide leads to the oxidization of vitamin B12, rendering it unusable in key enzymatic reactions necessary for myelin synthesis. Over time this qualitative deficiency leads to a central demyelination syndrome that characteristically develops despite normal serum vitamin B12 levels and, with continued exposure to nitrous oxide, resists treatment with vitamin B12 supplementation.

Method Nitrous oxide abusers presenting with a central demyelination syndrome were enrolled in this case series. Serum levels of vitamin B12, active B12, folate and homocysteine were measured. Nitrous oxide exposure was discontinued, and all patients were treated in accordance with evidence-based guidelines.

Results Eight patients presented with predominantly moderateto-severe clinical deficits. The majority were vitamin B12 replete. In most cases individuals had actively engaged in prolonged vitamin B12 supplementation in an attempt to circumvent the harmful pathophysiology, of which they were loosely aware. Following treatment and rehabilitation several patients were discharged into full-time care, and most had significant residual disability at follow-up.

Conclusions This case series not only illustrates the tragic consequences of abuse of this widely available and legally procured stimulant, but also highlights the futility of 